\title{
Prognostic Value of Lymphocyte Vascular Density and E-Cadherin in Inflammatory Breast Cancer
}

\author{
Paul H. Levine ${ }^{1}$, Heather J. Hoffman ${ }^{1}$, Audra MacNeil ${ }^{1}$, Salman Hashmi ${ }^{1}$, Sherry X. Yang ${ }^{2}$, \\ Stephen Hewitt ${ }^{3}$, Kenneth L. van Golen" ${ }^{4}$, Sandra M. Swain ${ }^{5}$ \\ ${ }^{1}$ Department of Epidemiology and Biostatistics, Milken Institute School of Public Health, The George \\ Washington University, Washington DC, USA \\ ${ }^{2}$ National Clinical Target Validation Laboratory, Division of Cancer Treatment \& Diagnosis, National Cancer \\ Institute, Bethesda, USA \\ ${ }^{3}$ Laboratory of Pathology, Center for Cancer Research, National Cancer Institute, Bethesda, USA \\ ${ }^{4}$ Department of Biological Sciences, Center for Translational Cancer Research, The University of Delaware, \\ Newark, USA \\ ${ }^{5}$ Washington Cancer Institute, MedStar Washington Hospital Center, Washington DC, USA \\ Email: * paulhlevine@earthlink.net
}

Received 19 September 2014; revised 15 October 2014; accepted 10 November 2014

Academic Editor: Tuhin Das, Moffitt Cancer Center, USA

Copyright (C) 2014 by authors and Scientific Research Publishing Inc.

This work is licensed under the Creative Commons Attribution International License (CC BY).

http://creativecommons.org/licenses/by/4.0/

(c) () Open Access

\section{Abstract}

Background: We recently evaluated four laboratory assays, vascular endothelial growth factor D (VEGF-D), E-cadherin, lymphatic vessel density (LVD) measured by podoplanin, and intra-lymphatic tumor emboli (ILTE), which showed notable differences between inflammatory breast cancer (IBC) and non-inflammatory locally advanced breast cancer (LABC). In this study we investigated the potential of the three most quantitatively measured markers, E-cadherin, LVD and VEGF-D, to predict survival in the IBC patients. Materials and Methods: This study involved the 100 cases identified in the Inflammatory Breast Cancer Registry (IBCR) whose tumors were previously evaluated for the four assays noted above. Living patients were recontacted and survival data were available for up to 17 years. Overall survival (OS) was analyzed through the Kaplan-Meier method stratified by E-cadherin, LVD, VEGF-D, and response to chemotherapy. The differences in OS curves were compared using the log-rank test. Results: The median OS for patients with high LVD was 6.63 years (95\% CI: 4.06 to 10.14), compared to median at 10 years not reached in those with low LVD $(p=0.03)$. There was a trend towards a longer median OS in patients with high E-cadherin (10.14, 95\% CI: 6.63 to 11.67), compared with those with low E-cadherin $(6.26,95 \%$ CI: 3.42 to undeterminable). VEGF-D levels showed no correlation with survival. Conclusion: Low LVD

\footnotetext{
"Corresponding author.
} 
significantly predicts better survival. High E-cadherin expression, as with non-IBC breast cancer and several other malignancies, tends to be associated with a better prognosis.

\author{
Keywords
}

IBC, Prognostic Markers, E-Cadherin, Lymphocyte Vascular Density

\title{
1. Introduction
}

Inflammatory breast cancer (IBC) is an unusual form of breast cancer marked by aggressiveness and biological behavior with a tendency to disseminate micrometastases from the outset rather than develop in a more controlled stepwise progression as seen in most carcinomas. Considered by some oncologists as a subgroup of locally advanced breast cancer (LABC) since they appear clinically similar, this appears to be misleading because LABC is usually the result of delay in diagnosis. LABC may not have the same extent of metastatic disease, has a better prognosis than IBC, and is declining in incidence while IBC appears to be increasing in incidence [1]. An important clinical feature of IBC is the marked response to chemotherapy, which may contribute to survival; the initial response to chemotherapy is an important predictor of long term survival [2]. Previous analysis of data from the IBC registry indicates that estrogen receptors and HER2-neu confer an advantage to the patient by allowing the long term use of therapy aimed at these particular receptors [2]. We recently evaluated several potential prognostic markers in a study assessing laboratory markers differentiating IBC from LABC, VEGF-D, E-cadherin, lymphatic vessel density (LVD) measured by podoplanin, and intra-lymphatic tumor emboli (ILTE) [3]. With the advantage of a long follow-up of the IBC patients in our IBC registry, we investigated the prognostic value of the three quantitative assays in determining long-term survival in IBC.

\section{Materials and Methods}

\subsection{Patients and Tumor Samples}

The details of the patient selection and laboratory assays utilized in this study have been reported previously [3]. Dates of patient observation ranged from July 25, 1991 (date of diagnosis for first patient) to September 21, 2011 (last date of follow-up for the long-term survivors). Briefly, 100 IBC patients were classified into two groups. A case was considered classic IBC if there was documentation of classical history and physical findings of erythema and peau d'orange involving the majority of the breast or histopathologic confirmation of dermal lymphatic invasion (DLI) that met the criteria for IBC diagnosis by the American Joint Committee on Cancer (AJCC) and/or the National Cancer Institute's Surveillance, Epidemiology, and End Results (SEER) Program. A group of atypical IBC cases with the clinical signs of IBC, also appearing acutely but differing from classic IBC because of involvement of less than half the breast [4], were compared with the classic IBC cases to investigate the validity of current case definitions. Approximately half of the samples examined were from pre-treatment biopsies and the other half were from post-chemotherapy mastectomies. Chemotherapy in all cases included at least three drugs, adriamycin in all cases and usually cyclophosphamide and a taxane. The doses varied according to individual physician preferences and patient responses.

\subsection{Immunohistochemical (IHC) Evaluation of Tumor Samples}

IHC staining of ER, podoplanin, E-cadherin and VEGF-D was performed on the IBC and non-IBC paraffinembedded tumor tissues using previously described methods [5] [6]. The antibodies used for ER, podoplanin and E-cadherin included mouse monoclonal anti-ER (clone 1D5, Dako Corporation), mouse monoclonal antipodoplanin (clone AB03, AngioBio Co.), and mouse monoclonal anti-E-cadherin (clone 36B5, Chemicon International Inc.). Immunochemistry for VEGF-D used anti-VEGF-D antibody (rabbit polyclonal SC-25784) from Santa Cruz Biotechnology at 1:100 after antigen retrieval at $\mathrm{pH}$ (in a pressure cooker for 20 minutes. The antigen-antibody reaction was detected by Dako Envision + detection sysrem and DAB.

\subsection{Determination of Response to Chemotherapy}

Clinical response to chemotherapy was categorized as complete, partial, or none. The response to chemotherapy 
was first categorized in the initial patient telephone interview and confirmed by review of the patient records (both by PHL) after the completion of chemotherapy. Patient response was defined as complete if she reported rapid resolution to normal breast size and appearance, usually before the second round of chemotherapy. Partial response was defined as a gradual but definite improvement over several courses of chemotherapy, with clinical manifestations minimal or absent at the time of mastectomy. No discordances were seen between the patient's and her clinician's reports.

\subsection{Statistical Analysis}

E-cadherin expression was quantitatively analyzed using an Automated Cellular Imaging System (ACIS) as previously described [6] [7]. Six areas of tumors were measured with a 40× scoring tool in ACIS. Staining Index (SI) was determined by staining intensity $\times \%$ staining per 100 . To validate the automated image analysis of E-cadherin, every 5th slide was read blindly by a pathologist and compared to the ACIS score. The scores were shown to be correlated, with a correlation coefficient of 0.501 .

Patients were categorized as having elevated or low E-cadherin levels with a level of 2.0 being the dividing line based on the distribution of the data, this value distinguishing between the two groups (Figure 1(a)). As shown in the histogram of LVD (Figure 1(b)), one-third of patients had a LVD value of zero. Patients were categorized as high LVD if they had levels greater than zero and low if they were equal to zero. Patients were categorized as having low or high VEGF-D levels based on the distribution of VEGF-D seen in the histogram (Figure 1(c)) with 145 chosen as the value to distinguish between the two groups.

Survival curves stratified by E-cadherin, LVD, and VEGF-D were estimated using the Kaplan-Meier method and compared for statistically significant differences using the log-rank test. Overall survival curves using the Kaplan-Meier method were estimated using stratification of response to chemotherapy and levels of E-cadherin and LVD. Since we had previously shown that response to chemotherapy was an important predictor of survival [2], E-cadherin and LVD were evaluated for predicting response to chemotherapy using a chi-square test and logistic regression. All statistical tests were 2-sided, and the level of significance was set at 0.05 . All statistical analyses were performed using SAS software, Version 9.2 (SAS Institute, Cary, NC).

\section{Results}

For all patients with available E-cadherin data $(\mathrm{n}=99)$, the median survival time was 8.39 years $(95 \%$ CI: 6.20 to 11.43). Approximately $50 \%$ of the IBC patients had a low E-cadherin level $(<2)$ comparable to levels of expression seen in non-IBC breast cancer [3] [8] (Figure 1(a)). The median survival time for patients with high E-cadherin levels $(n=52)$ was 10.14 years ( $95 \%$ CI: 6.63 to 11.67). The median survival time for patients with low E-cadherin levels $(n=47)$ was 6.26 (95\% CI was not calculable). A Kaplan-Meier survival curve for E-cadherin was not significant $(\mathrm{p}=0.24)$, though there was a distinct separation between high and low E-cadherin levels around year 10, in which the remaining sample size was greatly decreased (Figure 2(a)). A multiple comparison test of E-cadherin and response to treatment was statistically significant ( $p=0.016)$, so higher E-cadherin and better response to chemotherapy was associated with increased survival probability (Figure 2(b)). Of the few patients who had no response to chemotherapy, six patients had high E-cadherin levels and two patients had low E-cadherin levels.

LVD also appeared to show at least two populations of IBC patients (Figure 1(b)) with the highest number of patients being in the group with no detectable LVD greater than zero (low). The median survival time for patients with a low LVD $(n=33)$ appeared to be significantly greater than those with observable lymphatic vascular involvement $(n=66)$, the median survival for the latter group being 6.63 years (95\% CI: 4.06 to 10.14) whereas the group with median survival of the LVD zero group was not reached at 10 years. The Kaplan-Meier survival curve shows a statistically significant $(p=0.0288)$ separation between these two LVD groups, where low LVD is associated with better survival (Figure 3(a)). A multiple comparison test of LVD and response to chemotherapy was statistically significant $(p=0.017)$, so low LVD and better response to chemotherapy was associated with increased survival probability. A multiple comparison of E-cadherin and LVD was not statistically significantly associated with overall survival $(\mathrm{p}=0.12)$ (Figure 3(b)). There was a small sample of patients in some of the cells for analysis, specifically the none category for response to chemotherapy, with low LVD having three patients and high LVD having five patients.

For VEGF-D, no particular distribution pattern was observed (Figure 1(c)). There was not a significant 


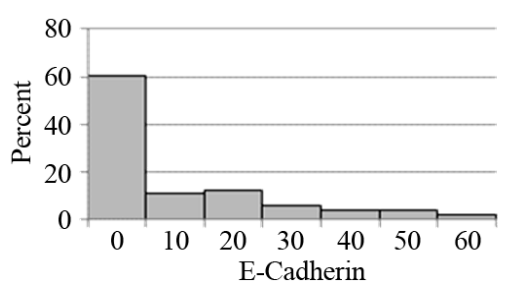

(a)

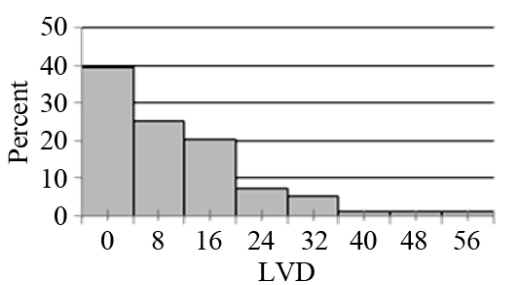

(b)

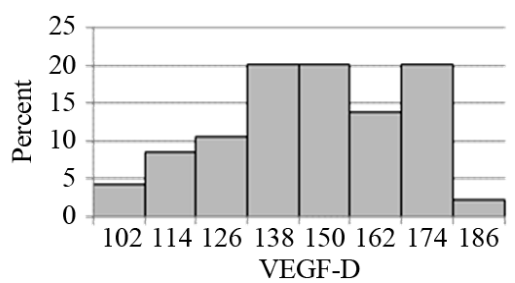

(c)

Figure 1. Histograms showing the distributions of (a) E-cadherin; (b) LVD; and (c) VEGF-D.

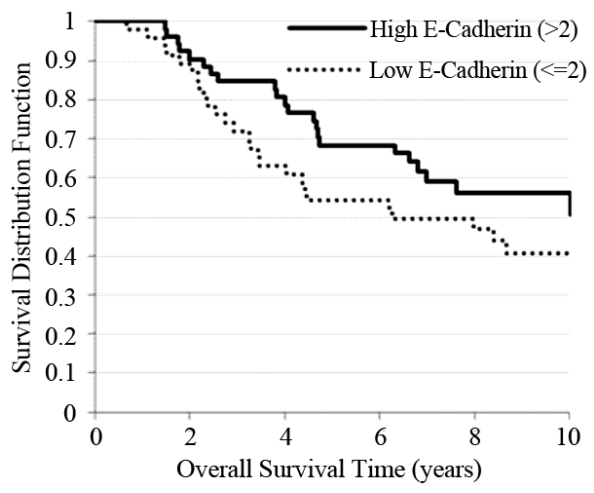

(a)

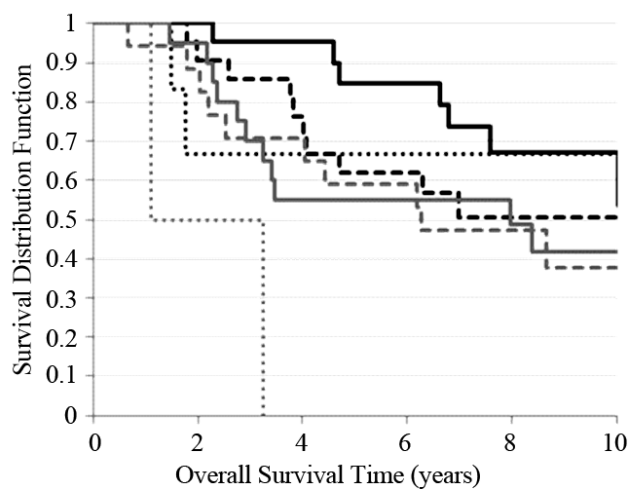

(b)

Figure 2. Kaplan-Meier survival curves stratified by (a) E-cadherin; and (b) E-cadherin and response to chemotherapy.

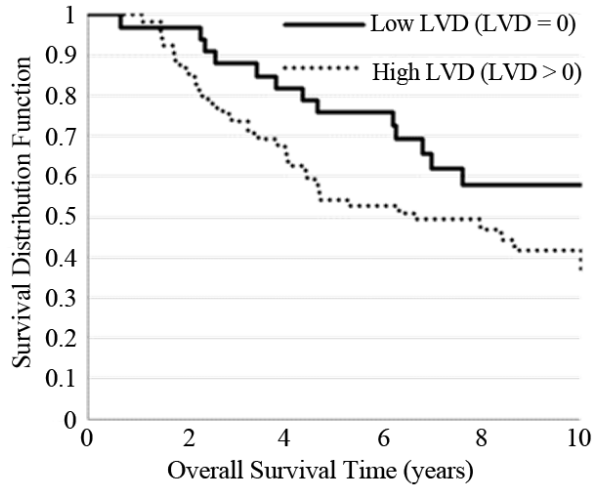

(a)

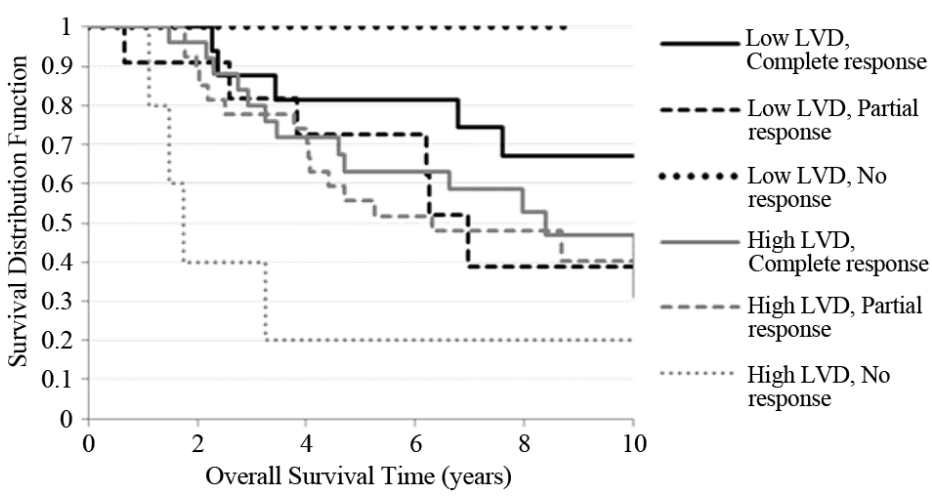

(b)

Figure 3. Kaplan-Meier survival curves stratified by (a) LVD; and (b) LVD and response to chemotherapy.

association ( $\mathrm{p}=0.39$ ) between VEGF-D and overall survival. We also saw no effect of the presence or absence of estrogen receptors on the prognostic value of LVD or E-cadherin.

E-cadherin $(p=0.32)$ and LVD $(p=0.54)$ did not predict response to chemotherapy.

\section{Discussion}

Inflammatory breast cancer (IBC) is a phenotypically distinct and unique form of breast cancer [8] [9]. IBC is arguably the most aggressive form of breast cancer and carries a guarded prognosis with 5- and 10-year diseasefree survival rates approaching $45 \%$ and $20 \%$, respectively. The morbidity of this disease is presumably due to the presence of tumor emboli in the dermal lymphatic vessels where they rapidly disseminate throughout the body [8]. Thus, the poor prognosis of IBC is due to extensive metastases to distant sites, particularly visceral organs [10]. IBC is clinically distinguished by rapid onset of primary skin changes, typically occurring within twelve weeks and progression to stage IIIb/IV disease within 6 months [8] [9] [11]. The clinical manifestation of 
IBC often resembles mastitis, presenting without a palpable mass, with primary IBC tumors presenting as diffuse sheets or cords of cells, which rapidly invade the dermal lymphatic vessels of the skin overlying the breast [8] [9] [11].

We evaluated three quantitative assays (LVD, E-cadherin and VEGF-D) found to differ between IBC and LABC in a previous study (3). Low LVD significantly predicted better survival, an expected finding as LVD is associated with IBC compared to non-inflammatory breast cancer and is associated with tumor aggressiveness (3).

E-cadherin, which has a molecular signature that is unique from other forms of breast cancer [12], also was associated with prognosis, high levels of expression correlating with a better prognosis. The cell adhesion molecule, E-cadherin, is highly expressed in a number of IBC cell lines and patient samples [13]-[16]. Conventionally, E-cadherin is thought of as a tumor suppressor and its loss contributes to tumor progression and metastasis [17]-[19]. Thus, the high level of E-cadherin expression in metastatic IBC is counter-intuitive.

It is suggested that IBC metastasizes via emboli spreading throughout the body by a process termed "passive metastasis" [20]. This hypothesis comes from a study where using palpation the intratumoral pressure of an IBC xenograft in a mouse model was increased, leading to detectable circulating tumor cells and increased pulmonary metastasis. However, studies with other types of tumors have demonstrated that palpation leads to increased shedding of tumor cells in the circulation [21]. Also, given the anatomy of the dermal lymphatics and the oscillatory flow of lymphatic fluid, it is unlikely that the patterns of IBC metastasis can be explained by emboli passively drifting through the lymphatics [22]-[24]. Data using an in vitro model of IBC emboli suggests that IBC cells continue to express E-cadherin while invading in a collective manner with motile cells being attached to one another [25]. Comparative studies of MARY-X, a human xenograft model of IBC with non-IBC xenografts indicated 10-20-fold overexpression of E-cadherin and MUC1, findings that were reflected in actual cases of human inflammatory breast cancer, and a $360^{\circ}$ circumferential distribution around the cancer cell membrane accounting for the formation of the lymphovascular embolus [14] [16] [26]. The lack of sialyl-Lewis X/A on MUC1 on MARY-X is suggested to account for the lack of tumor-endothelial cell attachment both in vitro and in vivo, which in turn facilitates metastasis [27]. Thus, it is speculated that a combination of high E-cadherin and MUC1 on IBC cells facilitates metastasis; however this model does not explain the rapid spread of IBC, nor does it explain the patterns of IBC dissemination counter-current to lymphatic flow.

Several studies in multiple tumor types suggest that E-cadherin expression correlates with disease progression and patient survival. A comparison of E-cadherin expression with classical histopathological features in bladder tumor specimens revealed that abnormal (negative or heterogenous) expression detected by immunohistochemistry correlated with both stage and grade and with tumor shape and size. Analysis of recurrence, progression and survival over a mean period of 36 months after surgery for bladder cancer showed that decreased E-cadherin immunoreactivity correlated strongly with poor outcome [28]. In another study, decreased E-cadherin expression was found to correlate with decreased myometrial invasion and was predictive for endometrial cancer mortality, disease progression, and extrapelvic recurrence, independent of known prognostic factors such as stage, grade, and histological subtype. There was a statistically significant decrease in overall survival (OS) in patients having tumors with decreased E-cadherin expression [29].

In a study of reduced E-cadherin expression in stage IV Wilms' tumor, heterozygous mutations were identified in the adhesion domain and intracellular portion of the E-cadherin. The study showed a significant association between E-cadherin expression and stage of disease with low expression corresponding to a 16.25 times increased risk for the presence of stage IV disease [30]. Investigation into the various aspects of E-cadherin biological activity, particularly in prostate cancer progression, revealed that the highly invasive, fibroblastic-like subpopulation of DU145 cells expressed less than 0.1-fold of E-cadherin protein when compared to the poorly invasive parental DU145 cells. An $80 \mathrm{kDa}$ E-cadherin fragment was detected, which on functional analyses revealed a stimulatory effect on the migratory and invasive capability of E-cadherin-positive cells [31]. In contrast to high E-cadherin expression in normal epithelia, ovarian surface epithelium exhibits some mesenchymal characteristics and contains little or no E-cadherin [32].

Prognostic indicators are important in cancer treatment as they may help group patients into subgroups requiring different types of treatment. Of four markers we evaluated to distinguish IBC from LABC [3], we investigated the prognostic value of the three most quantitative assays measuring LVD, VEGF-D and E-cadherin. In the present study we demonstrate that there are two identifiable populations of IBC patients that vary in their E-cadherin expression. The patients with tumor emboli with lower E-cadherin expression have a worse progno- 
sis than those with high E-cadherin expression. Interestingly, melanoma also undergoes lymphovascular invasion and there are reports of "inflammatory melanoma"; invasion of the dermal lymphatics by melanoma cells with a clinical presentation very similar to IBC [33]. Using the in vitro model of IBC emboli formation also studied a number of metastatic melanoma clones and demonstrated that although they formed tight emboli, did not express E-cadherin [25].

In a large study of invasive breast cancer (374 cases) of all stages, it was found that lymphatic microvessel density (LMVD) correlated with lymphatic vascular invasion (LVI) [34]. LMVD was associated more often with a risk of developing lymph node metastatses. However, in this study there was a significant decrease in patient's survival whose tumors had LVI $(p=0.0001)$ but not significant in those with LMVD $(p=0.78)$. Two other studies found that LMVD was significantly associated with lymph node metastatses and shorter survival in largely early stage breast cancer [35] [36]. In our study LVD low was associated with a significantly better survival, which is consistent with the two previously mentioned studies.

We hypothesize the possibility of phenotypic transition phases in the life of an IBC tumor cell or embolus where variable expression of different surface receptors either induce a "latent state", e.g. through a decrease in sialyl-Lewis X/A, thus inhibiting cell-matrix interactions and hence invasiveness [20], or an "active state". To define the active state we refer to a study [37], which showed that IBC SUM149 cells seeded in a media conditioned by human monocytes U937 cells, displayed motile phenotype, formed branched like structures associated with overexpression of fibronectin, whereas no alteration in the expression of E-cadherin was detected. IL-8 and MCP-1 were the major highly secreted cytokines by U937 cells [37], thus the role of tumor microenvironment in defining IBC features. Discussions within the IBC research community have focused on the thought that IBC cells are extremely plastic with regards to the epithelial to mesenchymal transition (EMT) [38]. It is suggested that IBC cells remain in an epithelial state but are primed to undergo EMT through co-expression of both epithelial and mesenchymal markers and then quickly revert back to an epithelial state. Our data suggests that there may be a population of IBC cells with low or no E-cadherin expression that are able to readily undergo EMT; however, this remains to be tested.

\section{Conclusion}

In summary, our data from the IBC registry indicate that the initial response to chemotherapy is the most important predictor of survival in IBC [2] but three other independent predictors of better prognosis are low LVD, high E-cadherin level and decreased diagnostic delay. One possible limitation to our laboratory data is the potential effect of chemotherapy on the various assays, but in our earlier study comparing E-cadherin, LVD, VEGF-D and ILTE in IBC vs. LABC [3] no difference was found between the patients whose tissues were pretreatment biopsies compared to those whose samples were post-chemotherapy mastectomies.

\section{References}

[1] Hance, K.W., Anderson, W.F., Devesa, S.S., Young, H.A. and Levine, P.H. (2005) Trends in Inflammatory Breast Carcinoma Incidence and Survival: The Surveillance, Epidemiology, and End Results Program at the National Cancer Institute. Journal of the National Cancer Institute, 97, 966-975. http://dx.doi.org/10.1093/jnci/dji172

[2] Hoffman, H.J., Khan, A., Ajmera, K.M., Zolfaghari, L., Schenfeld, J.R. and Levine, P.H. (2014) Initial Response to Chemotherapy, Not Delay in Diagnosis, Predicts Overall Survival in Inflammatory Breast Cancer Cases. American Journal of Clinical Oncology, 37, 315-321. http://dx.doi.org/10.1097/COC.0b013e318271b34b

[3] Levine, P.H., Portera, C.C., Hoffman, H.J., Yang, S.X., Takikita, M., Duong, Q.N., Hewitt, S.M. and Swain, S.M. (2012) Evaluation of Lymphangiogenic Factors, Vascular Endothelial Growth Factor D and E-Cadherin in Distinguishing Inflammatory from Locally Advanced Breast Cancer. Clinical Breast Cancer, 12, 232-239. http://dx.doi.org/10.1016/j.clbc.2012.04.005

[4] Levine, P.H., Zolfaghari, L., Young, H., Hafi, M., Cannon, T., Ganesan, C., Veneroso, C., Brem, R. and Sherman, M. (2010) What Is Inflammatory Breast Cancer? Revisiting the Case Definition. Cancers, 2, 143-152. http://dx.doi.org/10.3390/cancers2010143

[5] Van der Auwera, I., Van Laere, S.J., Van den Eynden, G.G., Benoy, I., van Dam, P., Colpaert, C.G., Fox, S.B., Turley, H., Harris, A.L., Van Marck, E.A., Vermeulen, P.B. and Dirix, L.Y. (2004) Increased Angiogenesis and Lymphangiogenesis in Inflammatory versus Noninflammatory Breast Cancer by Real-Time Reverse Transcriptase-PCR Gene Expression Quantification. Clinical Cancer Research, 10, 7965-7971. http://dx.doi.org/10.1158/1078-0432.CCR-04-0063

[6] Van der Auwera, I., Van den Eynden, G.G., Colpaert, C.G., Van Laere, S.J., van Dam, P., Van Marck, E.A., Dirix, L.Y. 
and Vermeulen, P.B. (2005) Tumor Lymphangiogenesis in Inflammatory Breast Carcinoma: A Histomorphometric Study. Clinical Cancer Research, 11, 7637-7642. http://dx.doi.org/10.1158/1078-0432.CCR-05-1142

[7] Tan, A.R., Yang, X., Hewitt, S.M., Berman, A., Lepper, E.R., Sparreboom, A., Parr, A.L., Figg, W.D., Chow, C., Steinberg, S.M., Bacharach, S.L., Whatley, M., et al. (2004) Evaluation of Biologic End Points and Pharmacokinetics in Patients with Metastatic Breast Cancer after Treatment with Erlotinib, an Epidermal Growth Factor Receptor Tyrosine Kinase Inhibitor. Journal of Clinical Oncology, 22, 3080-3090. http://dx.doi.org/10.1200/JCO.2004.08.189

[8] Kleer, C.G., van Golen, K.L. and Merajver, S.D. (2000) Molecular Biology of Breast Cancer Metastasis. Inflammatory Breast Cancer: Clinical Syndrome and Molecular Determinants. Breast Cancer Research, 2, 423-429. http://dx.doi.org/10.1186/bcr89

[9] Woodward, W.A. and Cristofanilli, M. (2009) Inflammatory Breast Cancer. Seminars in Radiation Oncology, 19, 256-265. http://dx.doi.org/10.1016/j.semradonc.2009.05.008

[10] Cristofanilli, M., Valero, V., Buzdar, A.U., Kau, S.W., Broglio, K.R., Gonzalez-Angulo, A.M., et al. (2007) Inflammatory Breast Cancer (IBC) and Patterns of Recurrence: Understanding the Biology of a Unique Disease. Cancer, 110, 1436-1444. http://dx.doi.org/10.1002/cncr.22927

[11] Jaiyesimi, I.A., Buzdar, A.U. and Hortobagyi, G. (1992) Inflammatory Breast Cancer: A Review. Journal of Clinical Oncology, 10, 1014-1024.

[12] Joglekar, M. and van Golen, K.L. (2012) Molecules That Drive the Invasion and Metastasis of Inflammatory Breast Cancer. In: Ueno, N.T. and Cristofanilli, M., Eds., Inflammatory Breast Cancer: An Update, Springer, New York, 161-184. http://dx.doi.org/10.1007/978-94-007-3907-9_15

[13] Ye, Y., Tellez, J.D., Durazo, M., Belcher, M., Yearsley, K. and Barsky, S.H. (2010) E-Cadherin Accumulation within the Lymphovascular Embolus of Inflammatory Breast Cancer Is Due to Altered Trafficking. Anticancer Research, 30 , 3903-3910.

[14] Kleer, C.G., van Golen, K.L., Braun, T. and Merajver, S.D. (2001) Persistent E-Cadherin Expression in Inflammatory Breast Cancer. Modern Pathology, 14, 458-464. http://dx.doi.org/10.1038/modpathol.3880334

[15] Tomlinson, J.S., Alpaugh, M.L. and Barsky, S.H. (2001) An Intact Overexpressed E-Cadherin/Alpha, Beta-Catenin Axis Characterizes the Lymphovascular Emboli of Inflammatory Breast Carcinoma. Cancer Research, 61, 5231-5241.

[16] Alpaugh, M.L., Tomlinson, J.S., Shao, Z.M. and Barsky, S.H. (1999) A Novel Human Xenograft Model of Inflammatory Breast Cancer. Cancer Research, 59, 5079-5084.

[17] Pignatelli, M., Ansari, T.W., Gunter, P., Liu, D., Hirano, S., Takeichi, M., et al. (1994) Loss of Membranous E-Cadherin Expression in Pancreatic Cancer: Correlation with Lymph Node Metastasis, High Grade, and Advanced Stage. The Journal of Pathology, 174, 243-248. http://dx.doi.org/10.1002/path.1711740403

[18] Perl, A.K., Wilgenbus, P., Dahl, U., Semb, H. and Christofori, G. (1998) A Causal Role for E-Cadherin in the Transition from Adenoma to Carcinoma. Nature, 392, 190-193. http://dx.doi.org/10.1038/32433

[19] Christofori, G. and Semb, H. (1999) The Role of the Cell-Adhesion Molecule E-Cadherin as a Tumour-Suppressor Gene. Trends in Biochemical Sciences, 24, 73-76. http://dx.doi.org/10.1016/S0968-0004(98)01343-7

[20] Alpaugh, M.L., Tomlinson, J.S., Kasraeian, S. and Barsky, S.H. (2002) Cooperative Role of E-Cadherin and SialylLewis X/A-Deficient MUC1 in the Passive Dissemination of Tumor Emboli in Inflammatory Breast Carcinoma. Oncogene, 21, 3631-3643. http://dx.doi.org/10.1038/sj.onc.1205389

[21] Price, J.E., Carr, D. and Tarin, D. (1984) Spontaneous and Induced Metastasis of Naturally Occurring Tumors in Mice: Analysis of Cell Shedding into the Blood. Journal of the National Cancer Institute, 73, 1319-1326.

[22] McMaster, P.D. (1947) The Relative Pressures with Cutaneous Lymphatic Capillaries and the Tissues. Journal of Experimental Medicine, 86, 293-308. http://dx.doi.org/10.1084/jem.86.4.293

[23] Daroczy, J. (1988) The Dermal Lympahtic Capillaries. Springer, New York. http://dx.doi.org/10.1007/978-3-642-73480-9

[24] Gray, H. (2000) The Lymphatic System. In: Lewis, W.H., Ed., Anatomy of the Human Body, 20th Edition, Bartleby, New York.

[25] Lehman, H.L., Dashner, E.J., Lucey, M., Vermeulen, P., Dirix, L., Van Laere, S. and van Golen, K.L. (2013) Modeling and Characterization of Inflammatory Breast Cancer Emboli Grown in Vitro. International Journal of Cancer, 132, 2283-2294. http://dx.doi.org/10.1002/ijc.27928

[26] Dong, H.M., Liu, G., Hou, Y.F., Wu, J., Lu, J.S., Luo, J.M., et al. (2007) Dominant-Negative E-Cadherin Inhibits the Invasiveness of Inflammatory Breast Cancer Cells in Vitro. Journal of Cancer Research and Clinical Oncology, 133, 83-92. http://dx.doi.org/10.1007/s00432-006-0140-6

[27] Alpaugh, M.L., Tomlinson, J.S., Ye, Y. and Barsky, S.H. (2002) Relationship of Sialyl-Lewis (X/A) Underexpression and E-Cadherin Overexpression in the Lymphovascular Embolus of Inflammatory Breast Carcinoma. The American 
Journal of Pathology, 161, 619-628. http://dx.doi.org/10.1016/S0002-9440(10)64217-4

[28] Popov, Z., Gil-Diez de Medina, S., Lefrere-Belda, M.A., Hoznek, A., Bastuji-Garin, S., Abbou, C.C., et al. (2000) Low E-Cadherin Expression in Bladder Cancer at the Transcriptional and Protein Level Provides Prognostic Information. British Journal of Cancer, 83, 209-214.

[29] Mell, L.K., Meyer, J.J., Tretiakova, M., Khramtsov, A., Gong, C., Yamada, S.D., et al. (2004) Prognostic Significance of E-Cadherin Protein Expression in Pathological Stage I-III Endometrial Cancer. Clinical Cancer Research, 10, 55465553. http://dx.doi.org/10.1158/1078-0432.CCR-0943-03

[30] Safford, S.D., Freemerman, A.J., Langdon, S., Bentley, R., Goyeau, D., Grundy, P.E., et al. (2005) Decreased E-Cadherin Expression Correlates with Higher Stage of Wilms’ Tumors. Journal of Pediatric Surgery, 40, 341-348. http://dx.doi.org/10.1016/j.jpedsurg.2004.10.030

[31] Chunthapong, J., Seftor, E.A., Khalkhali-Ellis, Z., Seftor, R.E., Amir, S., Lubaroff, D.M., et al. (2004) Dual Roles of E-Cadherin in Prostate Cancer Invasion. Journal of Cellular Biochemistry, 91, 649-661. http://dx.doi.org/10.1002/jcb.20032

[32] Auersperg, N., Pan, J., Grove, B.D., Peterson, T., Fisher, J., Maines-Bandiera, S., Somasiri, A. and Roskelley, C.D. (1999) E-Cadherin Induces Mesenchymal-to-Epithelial Transition in Human Ovarian Surface Epithelium. Proceedings of the National Academy of Sciences of the United States of America, 96, 6249-6254. http://dx.doi.org/10.1073/pnas.96.11.6249

[33] Haupt, H.M., Hood, A.F. and Cohen, M.H. (1984) Inflammatory Melanoma. Journal of the American Academy of Dermatology, 10, 52-55. http://dx.doi.org/10.1016/S0190-9622(84)80042-0

[34] Schoppmann, S.F., Bayer, G., Aumary, K., Taucher, S., Geleff, S., Rudas, K., Kubista, E., Hausmaninger, H., Samonigg, H., Gnant, M., Jakesz, R. and Horvat, R., Austrian Breast and Colorectal Cancer Study Group (2004) Prognostic Value of Lymphangiogenesis and Lymphovascular Invasion in Invasive Breast Cancer. Annals of Surgery, 240, 306312. http://dx.doi.org/10.1097/01.sla.0000133355.48672.22

[35] Nakumara, Y., Yasuoka, H., Tsujimoto, M., Imabun, S., Nakahara, M., Nakao, K., Nakamura, M., Mori, I. and Kakudo, K. (2005) Lymph Vessel Density Correlates with Nodal Status, VEGF-C Expression and Prognosis in Breast Cancer. Breast Cancer Research and Treatment, 91, 125-132. http://dx.doi.org/10.1007/s10549-004-5783-X

[36] Mohammed, R.A., Ellis, I.O., Eisheikh, S., Paish, E.C. and Martin, S.G. (2009) Lymphatic and Angiogenic Characteristics in Breast Cancer: Morphometric Analysis and Prognostic Implications. Breast Cancer Research and Treatment, 113, 261-273. http://dx.doi.org/10.1007/s10549-008-9936-1

[37] Mohamed, M.M. (2012) Monocytes Conditioned Media Stimulate Fibronectin Expression and Spreading of Inflammatory Breast Cancer Cells in Three-Dimensional Culture: A Mechanism Mediated by IL-8 Signaling Pathway. Cell Communication and Signaling, 10, 3. http://dx.doi.org/10.1186/1478-811X-10-3

[38] van Golen, K.L. and Cristofanilli, M. (2013) The Third International Inflammatory Breast Cancer Conference. Breast Cancer Research, 15, 318. 
Scientific Research Publishing (SCIRP) is one of the largest Open Access journal publishers. It is currently publishing more than 200 open access, online, peer-reviewed journals covering a wide range of academic disciplines. SCIRP serves the worldwide academic communities and contributes to the progress and application of science with its publication.

Other selected journals from SCIRP are listed as below. Submit your manuscript to us via either submit@scirp.org or Online Submission Portal.
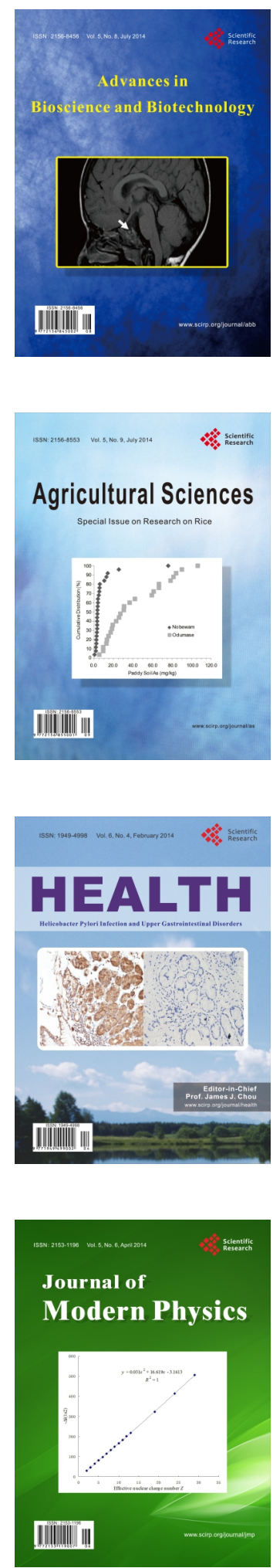
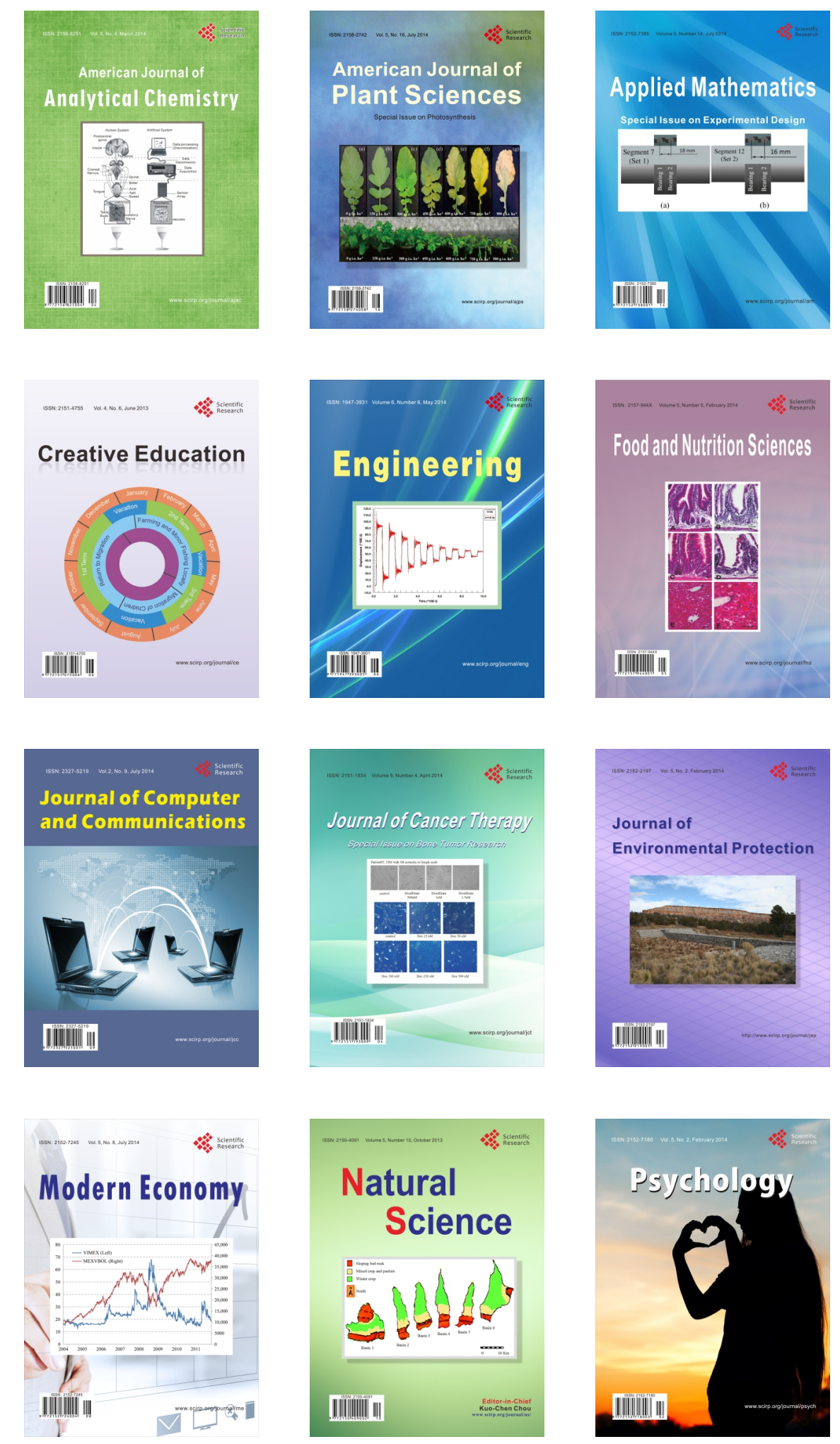